\title{
Early life insults as determinants of chronic obstructive pulmonary disease in adult life
}

This article was published in the following Dove Press journal:

International Journal of COPD

\author{
Osman Savran' \\ Charlotte Suppli Ulrik ${ }^{1,2}$ \\ 'Department of Respiratory Medicine, \\ Hvidovre Hospital, Hvidovre, \\ Denmark; ${ }^{2}$ Institute of Clinical \\ Medicine, University of Copenhagen, \\ Copenhagen, Denmark
}

Background: Early life events may predispose to the development of chronic lung disease in adulthood.

Aim: To provide an update on current knowledge of early nongenetic origins of COPD.

Materials and methods: Systematic literature review was performed according to the Preferred Reporting Items for Systematic Reviews and Meta-Analyses guidelines.

Results: A total of 16 studies, comprising 69,365 individuals, met the predefined criteria and were included in the present review. Studies have shown that in utero tobacco exposure, low birth weight, preterm birth, and respiratory diseases, primarily asthma and pneumonia, in early childhood are associated with lung function impairment later in childhood, and by that predispose to subsequent development of COPD, although the causal association between childhood respiratory diseases and COPD has been questioned in one study. Environmental tobacco exposure has also been shown to have negative impact on lung function in childhood possibly leading to COPD in adulthood, although it is at present not possible to clearly distinguish between the impact of active and the environmental tobacco exposure on subsequent development of COPD.

Conclusion: Tobacco exposure in utero and early life is a risk factor for subsequent development of COPD. Furthermore, low birth weight, lower respiratory tract infections and asthma, including wheezy bronchitis, in childhood also seem to be important determinants for later development of COPD. Early life insults may, therefore, be crucial to COPD development.

Keywords: COPD, respiratory infections, obstructive lung disease, preterm birth, tobacco exposure, early life, secondhand smoke

\section{Introduction}

COPD has for years been defined as chronic airflow obstruction caused by exposure to noxious particles or gasses, and in industrialized countries, COPD is generally regarded as a smoking-related disease. ${ }^{1-4}$ However, it is increasingly accepted that COPD can also be caused by other factors, including occupational exposure, and studies from recent years also suggest that COPD might in some cases have its origin in early life. ${ }^{5-9}$ In keeping with this, the 2017 update of the Global Initiative for Chronic Obstructive Lung Disease (GOLD) strategy document defines COPD as a disease characterized by persistent respiratory symptoms and airflow limitation usually, and by that not always, caused by exposure to noxious particles or gasses. ${ }^{3,10}$

COPD is the fourth leading cause of death globally and, at the individual level, COPD has substantial negative impact on quality of life. ${ }^{11,12}$ Reducing the incidence of COPD would substantially benefit overall health. Although focus in recent years has increasingly been on studies investigating the importance of early life events for subsequent development of COPD we have, so far, only limited knowledge of early life
Correspondence: Charlotte Suppli Ulrik Department of Respiratory Medicine, Hvidovre Hospital, Kettegård Alle 30, DK-2650 Hvidovre, Denmark Email csulrik@dadlnet.dk (c) (1) (5) 2018 Savran and Uliki. This work is published and licensed by Dove Medical Press Limited. The full terms of this license are available at https://www.dovepress.com/terms.php hereby accept the Terms. Non-commercial uses of the work are permitted without any furcher permission from Dove Medical Press Limited, provided the work is properly attributed. For permission for commercial use of this work, please see paragraphs 4.2 and 5 of our Terms (https://www.dovepress.com/terms.php). 
predictors of COPD, in spite of the fact that it may potentially have substantial impact on the future burden of morbidity and mortality caused by COPD. ${ }^{7,13-15}$ The aim of this review was, therefore, to provide an update on current knowledge regarding early life determinants for COPD.

\section{Materials and methods \\ Search strategy}

This systematic review was performed and reported in agreement with the Preferred Reporting Items for Systematic Reviews and Meta-Analyses guidelines. ${ }^{16}$ A systematic literature search was conducted (last updated November 2017) in the databases PubMed and EMBASE.

The search string for PubMed was as follows: (child OR child health OR children OR neonate OR prenatal OR preterm birth OR early) AND (COPD OR asthma OR obstructive lung disease OR nonreversible obstructive lung disease OR airflow limitation OR chronic obstructive respiratory disorder OR chronic respiratory disease) AND (adult OR young adult OR adult children) NOT medline [sb] OR ((“Genetic Predisposition to Disease"[Mesh] OR "Environmental Exposure"[Mesh] OR "Health Status Indicators"[Mesh] OR “adverse effects"[Subheading] OR "Long Term Adverse Effects"[Mesh] OR "Noxae"[Mesh] OR “Disease Progression"[Mesh] OR "Precipitating Factors"[Mesh] OR “Diet”[Mesh] OR “Smoking”[Mesh] OR “Allergens"[Mesh]) AND ("Child"[Mesh] OR "Infant”[Mesh] OR "Pregnancy”[Mesh]) AND ("Pulmonary Disease, Chronic Obstructive/epidemiology"[Mesh] OR "Pulmonary Disease, Chronic Obstructive/etiology"[Mesh] OR “Pulmonary Disease, Chronic Obstructive/prevention and control'"[Mesh])).

The search consisted of two parts: a free text search (the former part) and an MeSH term search (the latter part). The free text search retrieved all studies related to the stated search terms and the command "NOT medline [sb]" was included in order to identify potentially relevant studies that have not yet been indexed with MeSH terms.

The EMBASE search was conducted as follows: in the Ovid search database, the following search concept was entered: COPD. The term matched the following Subject Heading: chronic obstructive lung disease. Ovid MEDLINE automatically exploded (exp) the selected Subject Heading. Subheadings for "exp chronic obstructive lung disease" were chosen as "Etiology and Prevention." This resulted in "exp*chronic obstructive lung disease/et, pc [Etiology, Prevention]." The second search concept was "child" and the third search concept was "infant." The search was conducted by combining all three search concepts: "exp*chronic obstructive lung disease/et, pc [Etiology, Prevention] and child or infant."

Studies were included provided they fulfilled the following inclusion criteria: Original research that reported on exposure to potential early life predictors of COPD, and none of the exclusion criteria: Non-English publications and systematic reviews. The full paper of studies potentially fulfilling the prespecified criteria based on title and abstract were retrieved for further assessment (further details are given in Figure 1).

\section{Results}

A total of 137 publications potentially fulfilling the prespecified criteria were identified based on title and abstract.

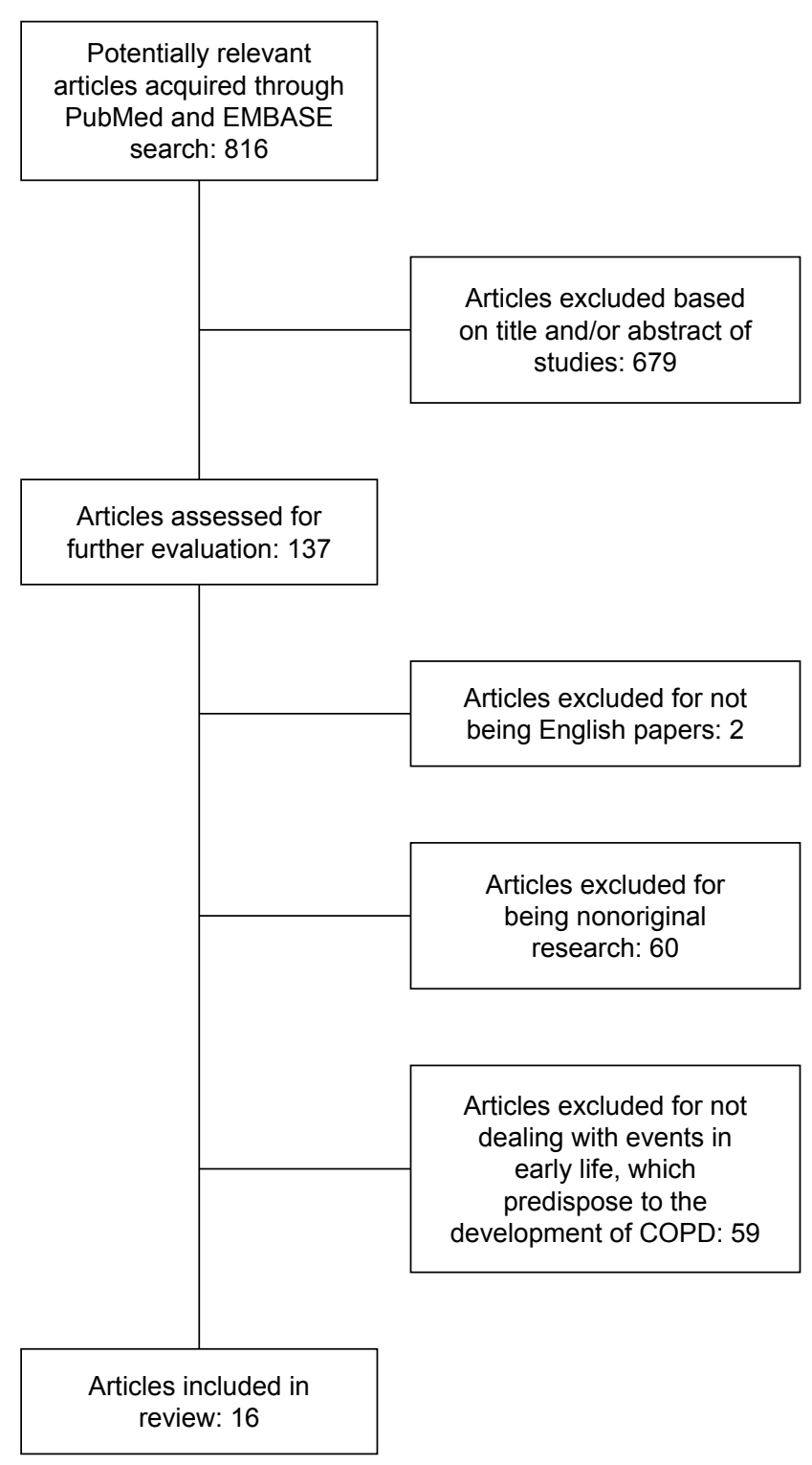

Figure I Consort diagram showing the study selection process. 
Sixteen studies comprising 69,365 individuals met the criteria based on full-text publications (Figure 1). Further details of the included studies are given in Table 1.

\section{Pre- and perinatal insults in early life Exposure to parental smoking in utero and childhood}

In a cross-sectional study published in 2006, De Sario et al investigated risk factors for different patterns of wheeze in 2107 Italian children aged 9-11 years. ${ }^{17}$ Based on onset of wheeze, the children were divided into four groups: early transient wheeze $(n=154,73 \%)$ (from birth to age 2 and no current wheeze), persistent wheeze $(n=51,2.4 \%)$ (from birth to age 2 and current wheeze), late onset wheeze ( $n=66$, $3.13 \%$ ) (current only), and control subjects ( $n=1,446,68.6 \%)$ (no early or current wheeze). Children with early transient wheeze had markedly reduced forced expiratory flow at $25 \%-75 \%$ of vital capacity $\left(\mathrm{FEF}_{25-75}\right)$ and the ratio of forced expiratory volume in 1 second to $\mathrm{FEV}_{1} / \mathrm{FVC}$. Furthermore, exposure to parental smoking both during pregnancy and during the child's first year of life was not only associated with persistent wheezing but also with reduced $\mathrm{FEF}_{25-75}$ and $\mathrm{FEV}_{1} / \mathrm{FVC}$ compared to control subjects. The study revealed an association between parental smoking and patterns of wheezing. ${ }^{17}$ However, as the parents of the participating children filled in the questionnaire in the study by De Sario et al, they may have over- or underreported the extent of the respiratory illnesses in their child, which may have biased the observed association between childhood wheeze and parental smoking, although it is difficult to think of an alternative way of sampling these data. ${ }^{17}$

In a cohort study published in 2016, Perret et al investigated whether maternal smoking predisposes offspring to later development of COPD. ${ }^{18}$ A total of 8,583 individuals from the population-based cohort Tasmanian Longitudinal Health Study (TAHS) were included. In short, TAHS consisted of Tasmanian schoolchildren born in 1961 and aged 7 years at the time of the first survey $(n=8,583)$. The aim of TAHS was to study asthma and environmental effects on asthma, allergy, and lung function. Subjects were followed up in 1968, 1974, 1991, and 2002. The baseline survey consisted of medical examination and spirometry, and of the 8,583 children who completed the survey, 93.5\% $(n=8,022)$ also completed lung function tests. In 1974 (aged 12), 7,380 (87.3\%) completed a follow-up survey. At the subsequent follow-up in 1991-1993 (mean age 30 years), 2,000 of the original cohort were selected based on their asthma status at baseline (1,000 with and 1,000 without asthma, respectively), of whom $86 \%$ were traced and sent a respiratory questionnaire on respiratory symptoms and lifestyle factors (smoking history and level of education). A total of 1,740 (78\%) of the original 2,000 subjects completed and returned the questionnaire. A follow-up was made in the year 2002 and included a postal survey, which was sent to 1,968 individuals from the original cohort, and a clinical study of a subsample of subjects. Some $88.1 \%$ of the 8,583 individuals were traced $(n=7,562)$ and $78.4 \%$ $(n=5,729)$ responded to a postal survey; $42 \%(n=2,387)$ of those who had responded to the postal survey were invited to participate in a clinical examination. These respondents were selected to enrich cases of asthma and cough reported in childhood or adulthood. Furthermore, postbronchodilator airflow obstruction was detected for $9.3 \%$ of all the middle-aged subjects. Subjects with mothers who had smoked heavily ( $>20$ cigarettes per day) were 2.7 times more likely to develop airway obstruction than subjects who had not been exposed to their mother's smoke. Among nonsmokers, any amount of maternal smoking was associated with lower static lung volumes, including total lung capacity, residual volume, and functional residual capacity. The study concluded that heavy parental smoking leads to obstructive lung disease in offspring. The study by Perret et al may, however, not give adequate evidence on whether smoke exposure from smoking mothers gives rise to COPD in patients. ${ }^{18}$ They did not account for the interference of air pollution in the development of COPD in subjects. It is well known that outdoor air pollution may cause COPD development over time. ${ }^{19}$ There were also difficulties in assessing exactly for how long and to what extent mothers had been smoking in the study by Perret et al. ${ }^{18}$

In a retrospective cohort study published in 2009 by Beyer et al, the long-term effects of secondhand smoke in childhood in patients with smoking-related COPD, defined according to GOLD was assessed. ${ }^{1,20}$ Information of lifetime tobacco exposure was obtained from all enrolled patients. The majority of smoking parents, ie, parents of the included COPD patients, had smoked more than 20 pack-years, and $15 \%$ of the mothers smoked regularly. Maternal smoking had a harmful impact on lung function in their offspring as COPD patients of smoking mothers had lower $\mathrm{FEV}_{1}$ than COPD patients, who were raised in households without tobacco exposure. Intrauterine exposure to tobacco smoke may have had an impact on the decreased $\mathrm{FEV}_{1}$ in COPD patients. ${ }^{20}$ The study revealed that maternal smoking decreased lung function in children. In addition, the authors of the study drew conclusions based on self-reported incidents dating back decades in the past, which makes the outcome more invalid and with high risk of recall bias. 


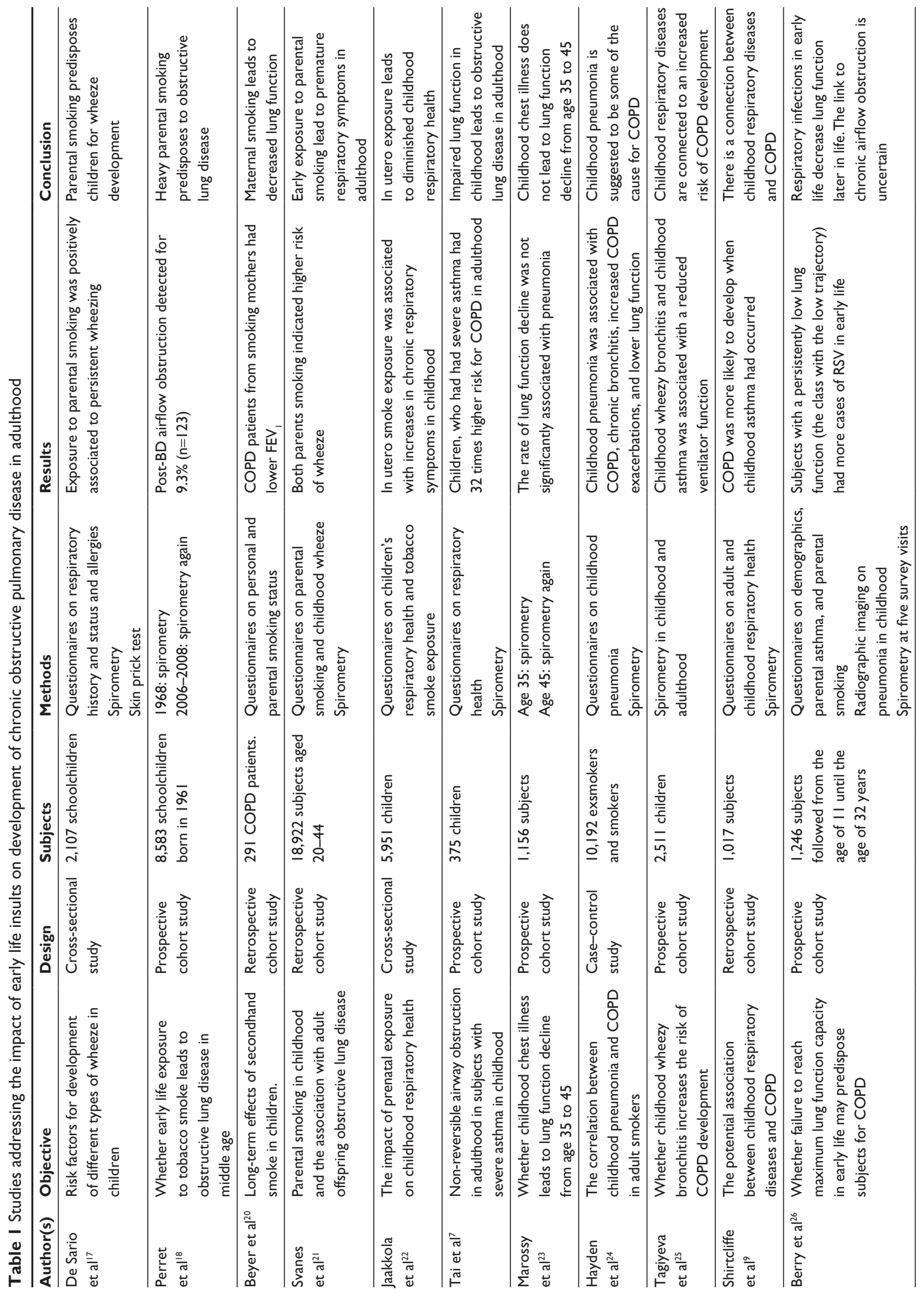




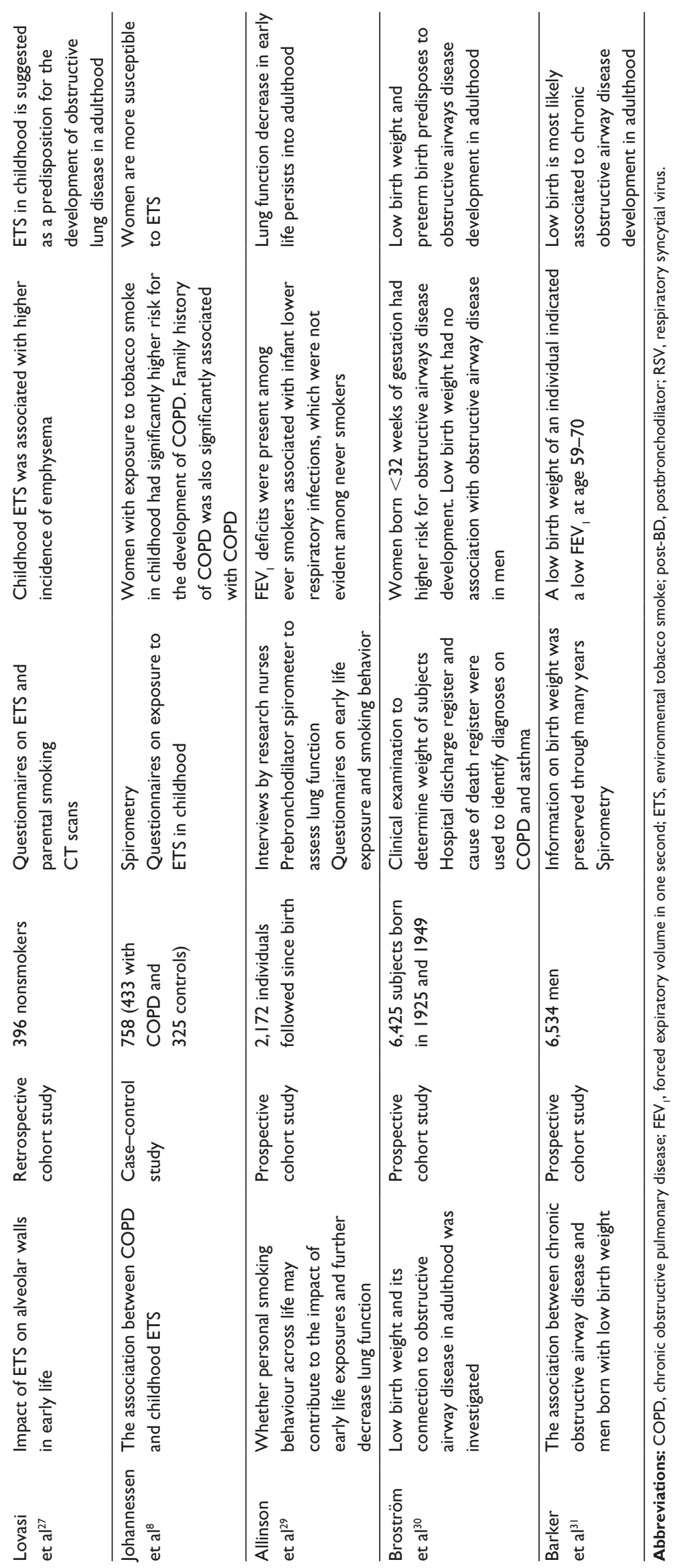


Based on retrospective information on childhood smoke exposure, the cohort study published in 2004 by Svanes et al examined the impact of parental smoking on wheeze in childhood and its association to adult obstructive lung disease. ${ }^{21}$ A series of questionnaires gathered information on adult smoking habits from 18,922 subjects, smokers and never smokers, between the age of 20 and 44. Spirometric data were available for 15,901 (84\%) subjects. Adult male individuals with fathers who smoked during their childhood had not only more respiratory symptoms, irrespective of their smoking status, but also lower $\mathrm{FEV}_{1} / \mathrm{FVC}$ ratio. Results indicated that wheeze in the childhood of subjects was more common when both parents had been smokers. However, similar to the previous study, the study demonstrates a disadvantage in retrieving information on subjects. Information recall, on which a diagnosis of childhood asthma or maternal smoking is based, means a high risk of recall bias. ${ }^{20}$

A cross-sectional study published in 2006 by Jaakkola et al examined possible lung function impairment related to intrauterine and postnatal tobacco exposure. ${ }^{22}$ The study population comprised 5,951 children aged 8 to 12 years. Parents were asked to fill in questionnaires on children's respiratory health and exposure to tobacco smoke. Estimation of tobacco exposure was based on self-reported information on maternal smoking during pregnancy, exposure to tobacco smoke during the first 2 years of life, after the age of 2 , and at the time of the survey obtained by questionnaire. The occurrence of low birth weight was higher among children of smoking women compared with children of nonsmoking women, and low birth weight increased the risk of asthma, asthma-like symptoms, and chronic respiratory symptoms. However, similar to the previous study, drawing conclusions based on incidents reported by parents may have biased the connection between parental smoking and decreased lung function in offspring. ${ }^{17}$

\section{Childhood respiratory disease}

The study by Tai et al published in 2014 was based on the prospective Melbourne asthma study cohort, and they investigated the association between severity of childhood asthma and adult COPD, defined as chronic airflow obstruction of children with asthma. ${ }^{7}$ Children were enrolled in the cohort at age 7 and it comprised 105 children with no wheezing (controls), 74 children with less than five episodes of wheezing associated with bronchitis, 113 children with wheezing not associated with respiratory infection, and 83 children with severe asthma (onset of symptoms before 3 years of age, persistent symptoms at 10 years of age, and $\mathrm{FEV}_{1} / \mathrm{FVC}<0.7$ at age 10). The subjects were regularly followed up, and at age 50, a total of 346 adults filled out questionnaires and had postbronchodilator spirometry performed. They were divided into four groups: subjects with no wheezing, those who originally had symptoms and went into remission, those with current asthma, and those with COPD (FEV ${ }_{1} /$ FVC ratio measured at age 50). Children, who had had severe asthma, had higher risk (odds ratio 32) for developing COPD in adulthood compared with controls. The study by Tai et al included a relatively small number of subjects, which may have led to selection bias and influenced the results.

In a prospective cohort study from 2006, Marossy et al investigated the effects of childhood chest illness on the rate of decline of lung function in subjects from the ages of $35-45$ years. ${ }^{23} \mathrm{~A}$ total of 1,156 subjects were from the British Cohort (National Child Development Study), which was a longitudinal study of all people in England, Scotland, and Wales born in the first week of March 1959. Individuals with a known early life respiratory infection and 293 control subjects (individuals with no history of these conditions matched according to age) from the cohort were included. Spirometry was performed at the age of 35 and repeated at 45 years. Multiple regression models, adjusted for sex, socioeconomic status at birth and at age 45 , smoking history, daily tobacco consumption age 35-45 years, month of test, observer, and lung function testing at each follow-up, were used to estimate the association between childhood chest illness and change in $\mathrm{FEV}_{1} / \mathrm{FVC}$ between ages 35 and 45 years for subjects and control subjects. Compared to control subjects, the rate of lung function decline was not significantly associated with respiratory conditions at age 7 . While the studies mostly agreed on the fact that childhood infections and asthma was connected to the development of COPD later in life, the study by Marossy et al disagreed., ${ }^{7,923-25}$ The authors acknowledged, however, that the findings could not rule out a decline in lung function above the age of 45 .

Based on a case-control study, Hayden et al recently investigated the association between childhood pneumonia and the presence of COPD in adult former and current smokers $(n=10,192) .{ }^{24} \mathrm{~A}$ total of $854(8.4 \%)$ subjects of the 10,192 individuals reported childhood pneumonia, and 405 (47\%) of the 854 subjects had COPD diagnosed by spirometry. Subjects with and without childhood pneumonia were compared by demographics, smoke exposure, and pneumonia history. A history of childhood pneumonia was associated with COPD, chronic bronchitis, COPD exacerbation rate, and lower lung function. The authors concluded that a history of childhood pneumonia combined with asthma substantially 
increased the risk of COPD. Furthermore, it is important to note that the strategy used for obtaining information in this study may have been subject to bias as individuals with a present diagnosis of obstructive lung disease may have been more likely to recall childhood illnesses.

The prospective cohort study by Tagiyeva et al published in 2015 investigated whether childhood wheezy bronchitis increased the risk of subsequent COPD. ${ }^{25}$ Of the cohort of children aged 10-15 years $(n=2,511), 121$ (4.8\%) and 167 (6.7\%) children, respectively, were classified as having childhood asthma and wheezy bronchitis, whereas the remaining 2,223 (89\%) children were controls. The cohort was recruited in 1964, and followed up in 1989, 1995, 2001, and 2014 at age $60-65$ years. Spirometry was performed at all surveys. Childhood wheezy bronchitis and childhood asthma was associated with an increased risk of COPD as defined by the GOLD (postbronchodilator $\mathrm{FEV}_{1} / \mathrm{FVC}<0.70$ ). ${ }^{1}$ The main limitation to this study was the restriction of the sample size, which occurred as the follow-up target was exceeded.

In 2012, Shirtcliffe et al published a retrospective study examining the association between age, sex, ethnicity, atopy, a diagnosis of childhood asthma, premature birth, early hospitalisation for lung diseases, and smoking and the development of COPD. ${ }^{9}$ A total of 3,500 adult individuals randomly selected from the cross-sectional Wellington Respiratory Survey were included. Subjects were divided according to age into the following groups: 25-39, 40-49, 50-59, 60-69, and 70+ years. A postal screening questionnaire was sent out to the subjects over a 12-month period (2003-2004). A total of 1,017 (29\%) replied, completed, and responded by returning the questionnaire. The questions were on premature birth $(<1$ month before the expected date of birth), early childhood hospitalizations, smoking history, and childhood asthma. Ethnicity was self-reported. A diagnosis of atopy was based on a positive skin prick test. A total of 749 of the 1,017 subjects $(77.9 \%)$ completed lung function tests, and of these, $116(15.4 \%)$ were diagnosed with COPD based on a postbronchodilator $\mathrm{FEV}_{1} / \mathrm{FVC}<0.7$. Of the 116 individuals with COPD, 22 (18.9\%) had a history of childhood asthma. The study concluded that the risk of COPD increased by 1.3 -fold per 10 pack-years of cigarettes, and the risk associated with childhood asthma corresponded to 62 pack-years of cigarette smoking. One particularly important limitation to this study was that other variables such as parental smoking or low socioeconomic status, which may have confounded the effects of childhood asthma on lung function, were not taken into account when risk of COPD was assessed.
The study by Berry et al published in 2016 investigated whether failure to gain maximal lung function in early life predisposed individuals for COPD later in life. ${ }^{26}$ The study used the Tucson Children's Respiratory Study cohort ( $n=1,246$ subjects) born between 1980 and 1984. Questionnaires were used to obtain information on demographics, parental asthma, and parental smoking. Radiographic imaging confirmed pneumonia in early life. Spirometry was performed at surveys conducted at ages 11, 16, 22, 26, and 32 years. Of the 1,246 subjects, 599 (48\%) had two or more spirometry measurements performed between age 11 and 32 years, for a total of 2,142 observations (119 with two observations, 166 with three observations, 164 with four observations, and 150 with five observations). A two-class model was used for the analysis of trajectories of lung function decline, as the two-class model presented a better fit for the analysis than the one-class model ( $p=0.0007)$. Among the 599 subjects in the two-group latent class analysis, $56(9.3 \%)$ were assigned to the persistently low lung function trajectory, which was defined as a probability greater than or equal to 0.5 . Of the 56 subjects assigned to the low trajectory class, most $(n=33 ; 58.9 \%)$ had a probability greater than or equal to 0.7 of belonging to their trajectory class. Similarly, among the 543 (91\%) subjects assigned to a normal trajectory class, most $(n=408 ; 75.1 \%)$ had a probability greater than or equal to 0.9 of belonging to the normal trajectory class. The persistently low trajectory class demonstrated an average absolute difference in $\mathrm{FEV}_{1} / \mathrm{FVC}$ ratio of $\sim 9 \%$ over the course of the 21-year follow-up. Subjects who had experienced lower respiratory infection caused by respiratory syncytial virus during the first 3 years of life was higher among the persistently low class compared with the normal class $(41.2 \%$ vs $21.4 \%$; $p=0.001)$. Subjects with a persistently low trajectory demonstrated lower lung function, which was measured by average maximal expiratory flow at functional residual capacity, during infancy and at age 6 years. The study concluded that the low lung function trajectory was likely an important pathway to COPD, as both those with rapid decline in lung function and those with lower initial lung function were more likely to reach the threshold level of lung function that is used to diagnose COPD compared with those with normal rate of decline in lung function. Furthermore, the study proposed a random method to define class membership for subjects as the authors decided that the probability had to be greater than or equal to 0.5 to define class membership. This led to a restriction in the number of included subjects and, therefore, may represent a limit to this study. 


\section{Environmental tobacco smoke (ETS)}

A retrospective cohort study by Lovasi et al published in 2010 investigated the potential damage caused by ETS on the alveolar walls in early life. ${ }^{27} \mathrm{~A}$ total of 1,781 subjects met the criteria for inclusion as they had lifetime tobacco exposure of less than 100 cigarettes (and by that were classified as never smokers), mean age 61 years and $65 \%$ were women. ${ }^{28}$ Signs of early emphysema were assessed by CT scans. Subjects, who had been exposed to cigarette smoke from smoking parents, had significantly more evidence of emphysema compared with subjects who did not have smoking parents. Still, there was a possibility that active smoking might have been additive to the damaging effect of ETS on the lungs, and therefore, compromised the results of the study.

In a further study published in 2012, Johannessen et al examined a possible association between COPD in adulthood and ETS in childhood in a case-control study. Subjects, who met the following criteria, were enrolled: COPD defined according to GOLD and tobacco exposure of more than 10 pack-years. ${ }^{1}$ The control group were from a previously sampled population, lived in the same area as COPD patients, and had a postbronchodilator $\mathrm{FEV}_{1}$ / $\mathrm{FVC} \geq 0.7$ and $\mathrm{FEV}_{1}>80 \%$ of predicted. Subjects included 433 COPD patients and a control group of 325 subjects aged 40-79 years. Information on childhood tobacco exposure was obtained by questionnaires, and the prevalence of childhood exposure to ETS was found to be $61 \%$. After adjusting for pack-years smoked and current smoking status, women with childhood tobacco exposure had a significantly higher risk of COPD compared to women who had not been exposed (an adjusted 1.9 times higher risk of COPD development). Exposure to dust or gas was significantly associated with COPD and all analyses were adjusted for smoking status, age, and pack-years smoked. A family history of COPD was also significantly associated with COPD in COPD patients. On the other hand, exposure to ETS was not found to be associated with the development of COPD in men. Although the available evidence suggests a direct causality between COPD and early life events, COPD in the patients with exposure to early life insults may in fact be COPD developed due to the extra pack-years of active smoking. ${ }^{8,20}$

In 2017, Allinson et al published a study investigating early life exposure to tobacco smoke and personal smoking and the development of permanent lung function. ${ }^{29}$ Data across seven decades from a nationally representative sample of 2,172 individuals were examined prospectively. Subjects were followed since their enrolment at birth in March 1946. The sample originally consisted of a nationally representative cohort called The Medical Research Council National Survey of Health and Development ( $\mathrm{n}=5,362$ subjects). Subjects were selected based on their representation of all single births to married women within England, Scotland, and Wales. At age 43 years, research nurses interviewed 3,632 individuals (68\%) at home. Loss to follow-up was either because subjects had died (7\%), emigrated (11\%), refused to participate (16\%) or were unable to be traced (5\%). Nurses made similar visits at ages 53 and at 60-64 years. Early life exposure was examined by asking questions on lower respiratory infections to parents, recording the number of occupants and rooms within the home (home overcrowding), social class (defined as either manual or nonmanual social class of the father of the included subjects), and pollution exposure during infancy of the cohort subjects. Prebronchodilator lung function was measured by spirometer at 43 years (mean 43.5; SD 0.2), 53 years (mean: 43.5; SD 0.2) and 60-64 years (mean 63.3; SD 1.1). Daily number of cigarettes smoked was recorded at age 20, 25, 36, 43,53 , and 60-64 years during nurse interviews or via postal questionnaires to estimate smoking behavior. In general, the findings indicated that $\mathrm{FEV}_{1}$ decline was found to be greater in smokers. Among the 2,172 individuals, however, there were $\mathrm{FEV}_{1}$ deficits associated with infant lower respiratory infection in ever smokers, which were not evident among never smokers. Additionally, a major limitation was the late implementation of spirometry measurements in the Medical Research Council National Survey of Health and Development. Guidelines for the protocol of this study were established before the use of spirometry, which may have led to an underrepresentation of cases with obstructive airway disease.

\section{Low birth weight}

In a prospective cohort study published in 2013, Broström et al examined the association between low birth weight and preterm birth and COPD. ${ }^{30}$ They followed up a cohort of infants born preterm ( $<35$ weeks) or with a low birth weight $(<2,000 \mathrm{~g}$ for girls and $<2,100 \mathrm{~g}$ for boys) in a population of 250,000 individuals from 1987 to 2006 . The population included both control subjects and infants born preterm and with a low birth weight. Asthma and COPD were defined from the Hospital Discharge Registers and Cause of Death Registers. Cohort subjects were considered cases if they had a main diagnosis of COPD or asthma with the belonging diagnostic codes. Results indicated that women, but not men, with low birth weight and/or gestational age had a significant increase in risk of obstructive airway disease. Results further indicated that women born before 32 weeks of gestation had 
a higher risk for asthma and COPD compared with full-term women (hazard ratio of 5.67 and 2.77, respectively).

The prospective cohort study published in 1991 by Barker et al examined whether low birth weight in subjects was associated with COPD development. ${ }^{31}$ In Hertfordshire from 1911 to 1930 , the attending health professionals, who saw children at home periodically, noted all births and infant weights. They also followed the children over time to examine the occurrence of illnesses. A total of 5,718 men born during this period were traced at the time of this study and their whereabouts were acknowledged. Most of the men born before 1923 had died and childhood illnesses were not recorded for this group of men. Of the men born during 1920-1930, 1,157 still lived in the countryside. Some 845 (73\%) men agreed to be visited, when asked if they wanted to participate in this study. Of the 5,718 men, 55 (almost 10\%) had died of COPD at follow-up around the age 59-70 years. Spirometry was performed with measurements of $\mathrm{FEV}_{1}$ and FVC. Birth weight was noted at birth. The results indicated that a decreased birth weight was significantly associated with a decreased $\mathrm{FEV}_{1}$ at ages 59-70. Furthermore, studies published years ago, including the study by Barker et al, might not be comparable to more recent studies due to different diagnostic criteria and methods. ${ }^{30,31}$ However, the observations reported by Barker et al seem plausible, not least because Broström et al confirmed the observations in the more recent study.

\section{Discussion}

This review of the currently available studies strongly suggests a strong association between early life insults and later development of COPD. So far, studies have been able to confirm that subjects with low lung function, caused by early life insults, are at higher risk of COPD compared to subjects with no history of low lung function in childhood. . $^{-9,24-26,31,32}$ The early life insults include exposure to parental smoking in utero and early life, ETS, childhood respiratory diseases such as asthma and infections, and children born with low birth weight. ${ }^{8,19,33-39}$

Studies have shown that COPD patients, who may have experienced early life events that decreased their lung functions, represent a distinct entity. ${ }^{7}$ The study emphasizes that childhood respiratory diseases are sufficient for the development of COPD. Thus, the conclusion is in-line with the 2017 definition of COPD in the GOLD strategy document. ${ }^{3}$ The definition embraces other factors that are included in this review and believed to be related to the development of COPD in adult life. However, while several of the included studies have followed groups of subjects over the course of many years, no studies have reported on the progression of COPD through its entire course. Thus, the present understanding of early life events, which may predispose individuals for COPD, is in many cases still incomplete.

In contrast to the presented studies, which merely reported on cases with low lung function development in adulthood due to early life insults, the study by Lange et al found that subjects had higher risk of COPD development once they reached adulthood compared with control subjects. ${ }^{17,18,21,22,40}$ The subjects had also obtained low lung function in early life, because of early life exposure to cigarette smoke, similar to the previously mentioned studies. ${ }^{17,18}$ The study by Lange et al, therefore, confirms the assumptions of these studies, which makes it very difficult to rule out the almost conclusive association between COPD and low lung function obtained in childhood due to prenatal and postnatal tobacco exposure from parents. However, although all the studies, which have been included in the present review, agree that lung function decline is critical in the development of obstructive airway disease, more research is still needed to explore the association between early life exposure to cigarette smoke and whether it permanently and irreversibly decreases lung function in children.

The recent study Lovasi et al does not report on a link between ETS and COPD, but rather a connection between emphysema and ETS. ${ }^{27}$ However, emphysema describes one of the structural abnormalities seen in COPD, which is chronic airflow obstruction caused by both small airway disease (obstructive bronchiolitis) and parenchymal destruction (emphysema). ${ }^{3}$ Evidence on whether early life exposure to ETS itself causes COPD later in life due to an irreversibly decreased lung function in children is still lacking. However, a possible connection between COPD and ETS is plausible as the findings reported by Lovasi et al are also reported in other studies. . $^{87,29}$

Similar to the study by Broström et al, the study by Kaczmarczyk et al examined whether preterm birth was a risk for the development of respiratory disease in later life. ${ }^{30,41}$ The results indicated, unlike the study by Broström et al, that there were no significant differences between preterm-born adults and the reference group of adults in terms of respiratory parameters. ${ }^{30}$ One limitation to the study by Kaczmarczyk et al was the relatively small number of subjects included in the study and that may be the reason why there were no significant findings in respiratory function in preterm-born individuals compared with healthy individuals. ${ }^{41}$ Whether preterm birth is also a risk factor for COPD, therefore, remains uncertain, because evidence on the association is not conclusive. 
The causality between early life risk factors and chronic lung disease presented in the present review represents an example of recent years of immense research and may give respiratory physicians an idea on what prevention strategies against COPD should encompass given that the disease is defined according to the most recent GOLD strategy document. Due to the vast amount of research on risk factors for chronic lung disease, which modifies lung function at different stages of life, the scope of the present review has been to provide an update on current knowledge regarding perinatal factors and childhood exposures as a determinant of chronic obstructive lung disease in adult life. It is of course clear that efforts to prevent the development of COPD must address not only perinatal factors and childhood exposures, although these are important risk factors, but should also focus on optimization of respiratory health both before conception, in utero and in the early years of life prevention of obesity, exposure to pollution, development of bronchopulmonary dysplasia, and impact of low socioeconomic status. ${ }^{19,42-45}$

In conclusion, the present review identified 16 studies addressing the impact of early life insults and adult COPD. Although conclusive evidence is still lacking, the available studies clearly suggest a strong association between COPD and early life events leading to lung function impairment in children. The most significant findings are that early life tobacco smoke exposure increases the risk of COPD, that maternal smoking decreases lung function in the offspring, and that susceptibility to ETS differs between genders. Additionally, low birth weight increases risk of obstructive lung disease in adulthood. More research on early life factors may lead to improved prevention strategies and by that reduce the future burden of COPD.

\section{Disclosure}

The authors report no conflicts of interest in this work.

\section{References}

1. Global Initiative for Chronic Obstructive Lung Disease [home page on the Internet]. Global strategy for the diagnosis, management, and prevention of chronic obstructive pulmonary disease [updated 2016]. Available from: http://goldcopd.org/global-strategy-diagnosis-management-preventioncopd-2016/

2. From the global strategy for the diagnosis $\mathrm{MaPoC}$, Global Initiative for Chronic Obstructive Lung Disease (GOLD) 2016. Available from: http:// goldcopd.org/

3. Vogelmeier CF, Criner GJ, Martinez FJ, et al. Global strategy for the diagnosis, management, and prevention of chronic obstructive lung disease 2017 report: GOLD executive summary. Eur Respir J. 2017;49(3):212-217.

4. Niewoehner DE. Clinical practice. Outpatient management of severe COPD. N Eng J Med. 2010;362(15):1407-1416.

5. Omland O, Wurtz ET, Aasen TB, et al. Occupational chronic obstructive pulmonary disease: a systematic literature review. Scand J Work Environ Health. 2014;40(1):19-35.
6. Kerkhof M, Boezen HM, Granell R, et al. Transient early wheeze and lung function in early childhood associated with chronic obstructive pulmonary disease genes. $J$ Allergy Clin Immunol. 2014;133(1): 68-76, e4.

7. Tai A, Tran H, Roberts M, Clarke N, Wilson J, Robertson CF. The association between childhood asthma and adult chronic obstructive pulmonary disease. Thorax. 2014;69(9):805-810.

8. Johannessen A, Bakke PS, Hardie JA, Eagan TM. Association of exposure to environmental tobacco smoke in childhood with chronic obstructive pulmonary disease and respiratory symptoms in adults. Respirology. 2012;17(3):499-505.

9. Shirtcliffe P, Marsh S, Travers J, Weatherall M, Beasley R. Childhood asthma and GOLD-defined chronic obstructive pulmonary disease. Internal Med J. 2012;42(1):83-88.

10. From the global strategy for the diagnosis $\mathrm{MaPoC}$, Global Initiative for Chronic Obstructive Lung Disease (GOLD) 2017. Available from: http://goldcopd.org

11. Postma DS, Bush A, van den Berge M. Risk factors and early origins of chronic obstructive pulmonary disease. Lancet. 2015;385(9971): 899-909.

12. Gore JM, Brophy CJ, Greenstone MA. How well do we care for patients with end stage chronic obstructive pulmonary disease (COPD)? A comparison of palliative care and quality of life in COPD and lung cancer. Thorax. 2000;55(12):1000-1006.

13. Canoy D, Pekkanen J, Elliott P, et al. Early growth and adult respiratory function in men and women followed from the fetal period to adulthood. Thorax. 2007;62(5):396-402.

14. Carraro S, Scheltema N, Bont L, Baraldi E. Early-life origins of chronic respiratory diseases: understanding and promoting healthy ageing. Eur Respir J. 2014;44(6):1682-1696.

15. Narang I, Bush A. Early origins of chronic obstructive pulmonary disease. Sem Fetal Neonat Med. 2012;17(2):112-118.

16. Liberati A, Altman DG, Tetzlaff J, et al. The PRISMA statement for reporting systematic reviews and meta-analyses of studies that evaluate health care interventions: explanation and elaboration. J Clin Epidemiol. 2009;62(10):e1-e34.

17. De Sario M, Di Domenicantonio R, Corbo G, et al. Characteristics of early transient, persistent, and late onset wheezers at 9 to 11 years of age. J Asthma. 2006;43(8):633-638.

18. Perret JL, Walters H, Johns D, et al. Mother's smoking and complex lung function of offspring in middle age: a cohort study from childhood. Respirology. 2016;21(5):911-919.

19. Grigg J. Particulate matter exposure in children: relevance to chronic obstructive pulmonary disease. Proc Am Thorac Soc. 2009;6(7): 564-569.

20. Beyer D, Mitfessel H, Gillissen A. Maternal smoking promotes chronic obstructive lung disease in the offspring as adults. Eur J Med Res. 2009; 14(Suppl 4):27-31.

21. Svanes C, Omenaas E, Jarvis D, Chinn S, Gulsvik A, Burney P. Parental smoking in childhood and adult obstructive lung disease: results from the European Community Respiratory Health Survey. Thorax. 2004;59(4): 295-302.

22. Jaakkola JJ, Kosheleva AA, Katsnelson BA, Kuzmin SV, Privalova LI, Spengler JD. Prenatal and postnatal tobacco smoke exposure and respiratory health in Russian children. Respir Res. 2006;7:48.

23. Marossy AE, Strachan DP, Rudnicka AR, Anderson HR. Childhood chest illness and the rate of decline of adult lung function between ages 35 and 45 years. Am J Respir Crit Care Med. 2007;175(4):355-359.

24. Hayden LP, Hobbs BD, Cohen RT, et al. Childhood pneumonia increases risk for chronic obstructive pulmonary disease: the COPDGene study. Respir Res. 2015;16:115.

25. Tagiyeva N, Devereux G, Fielding S, Turner S, Douglas G. Outcomes of childhood asthma and wheezy bronchitis. A 50-year cohort study. Am J Respir Crit Care Med. 2016;193(1):23-30.

26. Berry CE, Billheimer D, Jenkins IC, et al. A distinct low lung function trajectory from childhood to the fourth decade of life. Am J Respir Crit Care Med. 2016;194(5):607-612. 
27. Lovasi GS, Diez Roux AV, Hoffman EA, Kawut SM, Jacobs DR Jr, Barr RG. Association of environmental tobacco smoke exposure in childhood with early emphysema in adulthood among nonsmokers. The MESA-lung study. Am J Epidemiol. 2010;171(1):54-62.

28. Centers for Disease Control and Prevention (CDC) [home page on the Internet]. National Health Interview Surevy. Available from: https:// www.cdc.gov/nchs/nhis/tobacco/tobacco_glossary.htm

29. Allinson JP, Hardy R, Donaldson GC, Shaheen SO, Kuh D, Wedzicha JA. Combined impact of smoking and early life exposures on adult lung function trajectories. Am J Respir Crit Care Med. 2017;196(8):11021-1030.

30. Broström EB, Akre O, Katz-Salamon M, Jaraj D, Kaijser M. Obstructive pulmonary disease in old age among individuals born preterm. Eur $J$ Epidemiol. 2013;28(1):79-85.

31. Barker DJ, Godfrey KM, Fall C, Osmond C, Winter PD, Shaheen SO. Relation of birth weight and childhood respiratory infection to adult lung function and death from chronic obstructive airways disease. $B M J$. 1991;303(6804):671-675.

32. Broström EB, Thunqvist P, Adenfelt G, Borling E, Katz-Salamon M. Obstructive lung disease in children with mild to severe BPD. Respir Med. 2010;104(3):362-370.

33. Hylkema MN, Sterk PJ, de Boer WI, Postma DS. Tobacco use in relation to COPD and asthma. Eur Respir J. 2007;29(3):438-445.

34. Hylkema MN, Blacquiere MJ. Intrauterine effects of maternal smoking on sensitization, asthma, and chronic obstructive pulmonary disease. Proc Am Thorac Soc. 2009;6(8):660-662.

35. Gilliland FD, Berhane K, McConnell R, et al. Maternal smoking during pregnancy, environmental tobacco smoke exposure and childhood lung function. Thorax. 2000;55(4):271-276.

36. Halvorsen T, Skadberg BT, Eide GE, Roksund OD, Carlsen KH, Bakke P. Pulmonary outcome in adolescents of extreme preterm birth: a regional cohort study. Acta Paediatr. 2004;93(10):1294-1300.
37. Hart SM, McNair M, Gamsu HR, Price JF. Pulmonary interstitial emphysema in very low birthweight infants. Arch Dis Child. 1983;58(8): 612-615.

38. Diaz AA, Hardin ME, Come CE, et al. Childhood-onset asthma in smokers. Association between CT measures of airway size, lung function, and chronic airflow obstruction. Ann Am Thorac Soc. 2014;11(9): 1371-1378.

39. Chan JY, Stern DA, Guerra S, Wright AL, Morgan WJ, Martinez FD. Pneumonia in childhood and impaired lung function in adults: a longitudinal study. Pediatrics. 2015;135(4):607-616.

40. Lange P, Celli B, Agusti A, et al. Lung-function trajectories leading to chronic obstructive pulmonary disease. New Eng J Med. 2015;373(2): $111-122$.

41. Kaczmarczyk K, Wiszomirska I, Szturmowicz M, Magiera A, Blazkiewicz M. Are preterm-born survivors at risk of long-term respiratory disease? Therap Adv Respir Dis. 2017;11(7):277-287.

42. Bhandari A, McGrath-Morrow S. Long-term pulmonary outcomes of patients with bronchopulmonary dysplasia. Semin Perinatol. 2013;37(2): $132-137$.

43. Griffiths PS, Walton C, Samsell L, Perez MK, Piedimonte G. Maternal high-fat hypercaloric diet during pregnancy results in persistent metabolic and respiratory abnormalities in offspring. Pediatr Res. 2016; 79(2):278-286.

44. Menezes AM, Dumith SC, Perez-Padilla R, et al. Socioeconomic trajectory from birth to adolescence and lung function: prospective birth cohort study. BMC Public Health. 2011;11:596.

45. Hemminki K, Li X, Sundquist K, Sundquist J. Familial risks for chronic obstructive pulmonary disease among siblings based on hospitalisations in Sweden. J Epidemiol Community Health. 2008;62(5):398-401.
International Journal of COPD

\section{Publish your work in this journal}

The International Journal of COPD is an international, peer-reviewed journal of therapeutics and pharmacology focusing on concise rapid reporting of clinical studies and reviews in COPD. Special focus is given to the pathophysiological processes underlying the disease, intervention programs, patient focused education, and self management protocols.

\section{Dovepress}

This journal is indexed on PubMed Central, MedLine and CAS. The manuscript management system is completely online and includes a very quick and fair peer-review system, which is all easy to use. Visit http://www.dovepress.com/testimonials.php to read real quotes from published authors. 